\title{
Tomography, Particle Physics and Medical Engineering Based on Waves as an Ultra-Modern Multidisciplinary Medical
}

\author{
Seyed Hossein Abrehdari* \\ Physics Department of Amirkabir University of Technology \& Institute of Geophysics of University of Tehran, Iran
}

*Corresponding author: Seyed Hossein Abrehdari, Physics Department of Amirkabir University of Technology \& Institute of Geophysics of University of Tehran; Iran, Institute of Geophysics and Seismic Engineering, National Academy of Science, Armenia

\begin{abstract}
ARTICLE INFO
Received: 㓞 February 21, 2019

Published: March 01, 2019

Citation: Seyed Hossein Abrehdari. Tomography, Particle Physics and Medical Engineering Based on Waves as an Ultra-Modern Multidisciplinary Medical. Biomed J Sci \& Tech Res 15(3)-2019. BJSTR. MS.ID.002700.

Abbreviations: EEG: Electroencephalogram; CT: Computed Tomography; BME: Biomedical Engineering; MRI: Magnetic Resonance Imaging

ABSTRACT

Imaging from inside the Human Body, the Earth and so on, with using the electromagnetic waves propagation/signals, tomography and particle physics is an emerging and growing technology in the advanced scientific and research centres of the world. In fact, magical images from inside the human body in the medical world, very briefly, has produced by carrying energy from wave particles or photons in tomography and particle physics. This beneficial technique has been used in Medical Engineering methods such as elastography, MRI, CT-scan, electroencephalogram (EEG), X-ray, echograph, optical tomography and many other beneficial techniques. Applications of these waves in the medical world don't have any mean without tomography interpretation or Interpretation of CT images computerized (computed tomography). Therefore, Detection and determination hot and cold spots in this kind images and how to spread the wave velocity propagation in hard and soft areas and tissues, is the most important step in identifying diseases and injuries in the medical world. In the following, some uses of these wave particles in medical world, briefly (mini- review) is mentioned.
\end{abstract}

Keywords: Medical Engineering; Wave particles; Tomography; Particle Physics; Ultramodern Multidisciplinary Medical; Diathermia; Hyperthermia

\section{Introduction}

All three methods are based on waves, amplitude, rhythm and frequency oscillations in the human body or the earth structure. Geophysical Tomography Method (using surface waves to understand and image the Earth) and Astroparticle Physics (using particles physics to understand and image phenomena) are interesting methods for understanding and image inside the earth, human body and etcetera. Tomography is imaging by sections or sectioning, through the use of any kind of penetrating wave. The method is used in radiology, archaeology, biology, atmospheric science, geophysics, oceanography, plasma physics, materials science, astrophysics, quantum information, and other areas of science. A device used in tomography is called a tomograph, while the image produced is a tomogram (Figure 1). Particle physics, is an inter disciplinary and young research field, grown at the intersection of particle physics, nuclear physics, astrophysics and cosmology. It addresses some of the most pressing and fundamental questions in science today: the origin and the evolution of our universe, the nature of dark matter and dark energy, the study of neutrinos and of the ultimate constituents of matter, the search for gravitational waves, the investigation and explanation of the cosmic rays (Figure 2). Biomedical Engineering (BME) or Medical Engineering is the application of engineering principles and design concepts to medicine and biology for healthcare purposes (e.g. diagnostic or therapeutic). This field seeks to close the gap between engineering and medicine, combining the design and problem-solving skills of engineering with medical biological sciences to advance health care treatment, including diagnosis, monitoring, and therapy. 


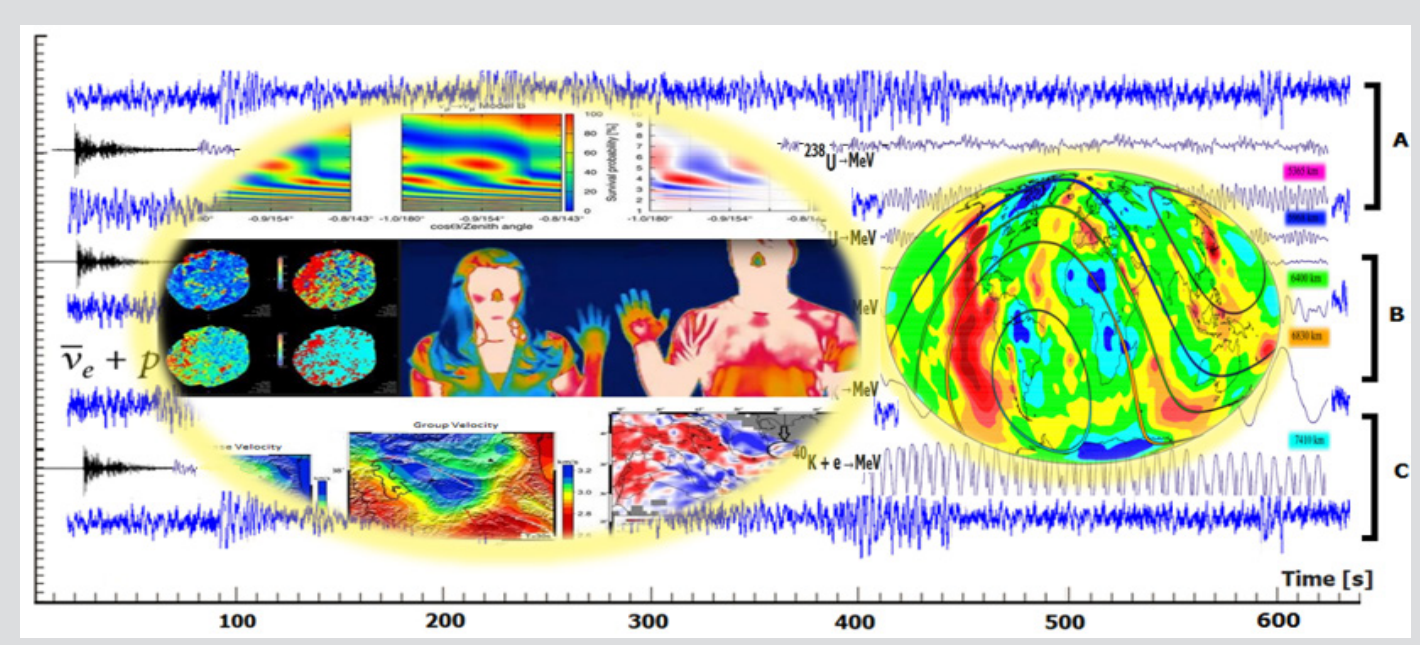

Figure 1: Samples of tomography and particle physics (part A and C). Well seen that interpreting and comparing them are possible based on an investigation of velocity structure, group velocity for different periods and high or low velocity anomalies $[4,6,7,18]$. Medical engineering (B images) record and interpret changes that occur at the surface temperature of the body and distribution of geothermal power density on the Earth $[10,11,18]$. (the images are slightly changed and designed by Abrehdari).

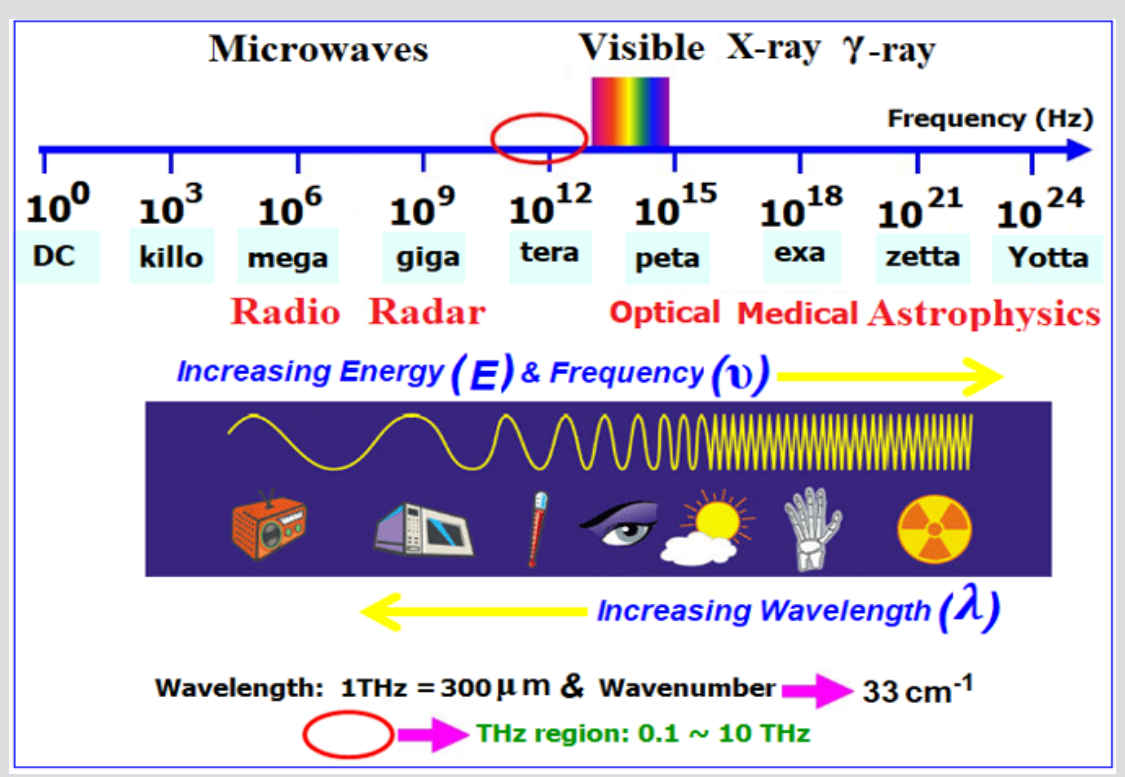

Figure 2: Different wavelengths of electromagnetic waves $[17,18]$. (the image is slightly changed and designed by Abrehdari).

\section{Application of waves in the medical world}

In various fields of medicine, from electromagnetic waves is used. In the following, some of the applications of these waves in summarized has been said.

\section{Diathermia}

Diathermy is one of the topics of the medical engineering branch and its topic is the heating of the internal tissues of the human body using electromagnetic radiation. Diathermy is divided into two parts: 1- short wave 2- microwave waves. In short-wave diathermy, electromagnetic waves with frequencies of $\mathrm{MHz}$ magnitude (13.56 or 27.12 MHz) are used (see Figure 1).

\section{Hyperthermia}

As mentioned, one of the applications of electromagnetic waves is create hyperthermia in the human body tissue. Hyperthermia with using electromagnetic waves and heat creation in damaged tissues, can help to treat cancers, chemotherapy, and radiation therapy. Also, it can increase the cancer cells' sensitivity to many treatments or the effect of anticancer drugs. Today, Hyperthermia because of reducing the amount of fear and the attacking pain and death rate and with regard to the development of tools and techniques, it has attracted the attention of many researchers (Figure 3). Types of hyperthermia included: 


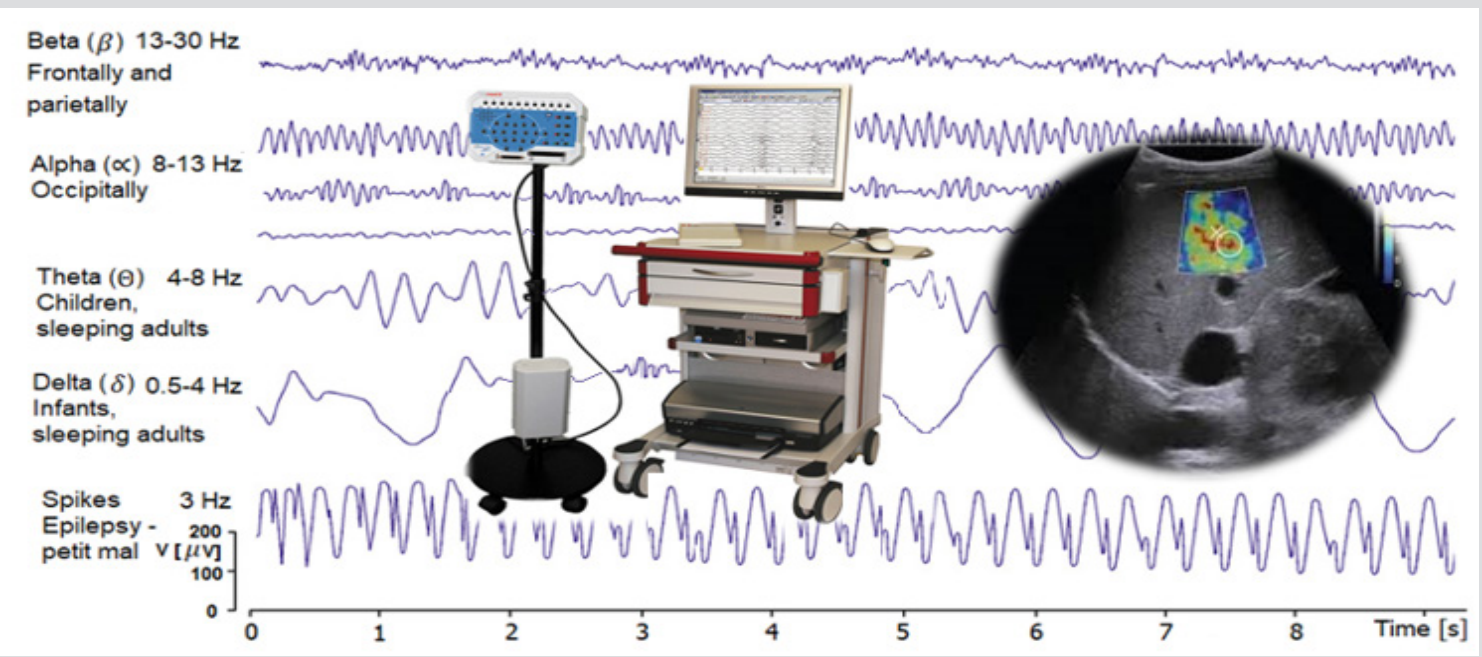

Figure 3: A picture of the medical engineering proceeds [17], (the images are slightly changed and designed by Abrehdari).

a) Topical hyperthermia involves warming up a very small area (usually Tumor)

b) Regional hyperthermia included heating a wide area (an organ)

c) Total hyperthermia; heat up the human body to a temperature of about 39 to 41 degrees centigrade.

In the use of hyperthermia for the treatment of cancers, though several reports have shown that burns occur at high levels and underlying layers (!!burning skin of the victims of the Bam earthquake in Iran has reported by the local people. Also, Earthquake waves are rich in phases and different frequencies!!).

\section{Other Applications of Heat Therapy by Waves}

Heat generated in the tissues, due to absorption of waves radiation, it can have other therapeutic effects that let us briefly mention them:

a. Enhance the body immunity with high-temperature via (e.g. to control AIDS)

b. Magnetic resonance imaging (MRI)

c. Restoration and proliferation of neural cells

d. Restoration of cartilage and swollen tissues and injuries

e. Electromagnetic knives

f. Destroying cancerous tissues relatively deep areas of the body

g. Pain control

h. Telemetry and etc.

For prefect information and knowledge about tomography and particle physics; see [1-16] references. Eventually we realized that these three important methods, namely medical physics, wave tomography and particle physics work according to the intensity of radiation, some subjects such as high frequency currents, fluctuations, oscillating devices, frequency oscillations, oscillation damping, energy transfer between oscillating devices, radio receivers and oscillatory currents have been carefully studied. The images produced by these methods are based on the intensity and light darkness (weakness); in other words, we have guided a spectrum of beams and photons (Figure 4). It really can be attractive and ultra-modern multidisciplinary medicine and in fact, we will take a step toward Molecular Imaging. Molecular imaging originated from the field of radiopharmacology due to the need to better understand fundamental molecular pathways inside organisms in a noninvasive manner (see Figure 4).

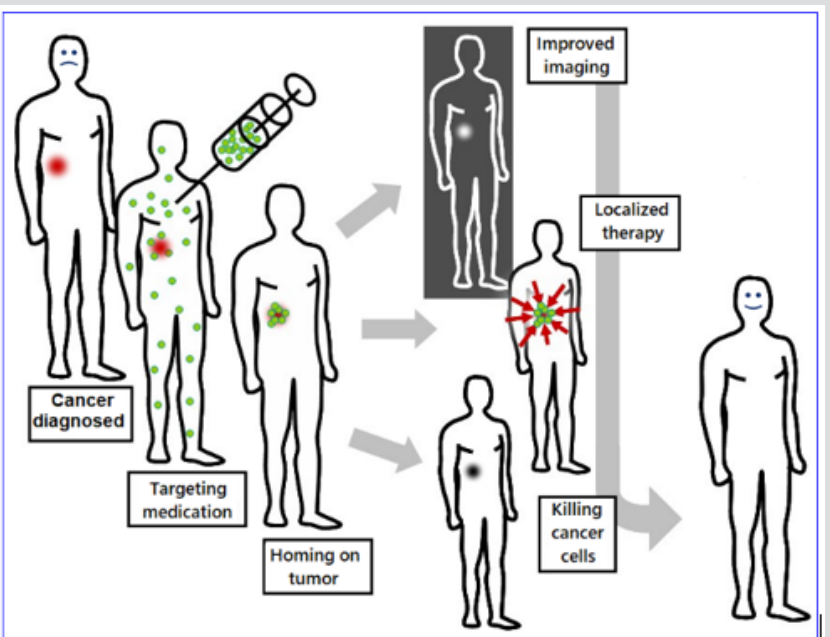

Figure 4: Molecular imaging \& therapy [19], (the image is slightly changed and designed by Abrehdari).

\section{References}

1. C Rott, A Taketa, D Bose (2015) Spectrometry of the Earth using Neutrino Oscillations. Scientific Reports 5: 15225.

2. WalterWinter (2016) Atmospheric neutrino oscillations for Earth tomography. Nuclear Physics B 908: 250-267. 
3. Walter Winter (2016) Atmospheric Neutrino Oscillations for Earth Tomography. Phenomenology.

4. Walter Winter DESY, Zeuthen (2016) ISAPP summer institute: Using particle physics to understand and image the earth GSSI, L'Aquila, Italy July p. 11-21.

5. Hiroko Watanabe (2016) Directionality in neutrino detection Research Center for Neutrino Science, Tohoku University \& ISAPP summer institute: Using particle physics to understand and image the earth GSSI, L'Aquila, Italy July p. 11-21.

6. H Zandi, H Rahimi (2016) Two-dimensional Rayleigh wave group velocity tomography in Northwestern part of Iranian Plateau. Jornal of earth and space physics 42(2): 221-232.

7. Cecil H, Ida M Green Institute of Geophysics and Planetary Physics is located in La Jolla, and is a Division of SIO.

8. G Di Molfetta, A Pérez (2016) Quantum walks as simulators of neutrinooscillations in a vacuum and matter. New Journal of Physics.

9. Hamza VM, Cardoso RR, Ponte Neto CF (2008) Spherical harmonic analysis of Earth's con ductive heat flow. Int J of Earth Sciences 97(2): 205-226.

10. VD Rusov, DA Litvinov, S Cht Mavrodiev, EP Linnik, VN Vaschenco, et al. (2010) Vaschenko Kamland-experiment and soliton-like nuclear georeactorpart 1. Comparison of theory with experiment, p. 1-30.

\section{ISSN: 2574-1241}

\section{DOI: 10.26717/BJSTR.2019.15.002700}

Seyed Hossein Abrehdari. Biomed J Sci \& Tech Res

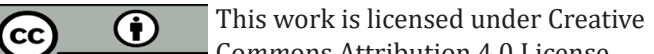
Commons Attribution 4.0 License

Submission Link: https://biomedres.us/submit-manuscript.php
11. P Alivisatos, S Berridge, N Bokor, C Britton, W Brayn (1998) A Liquid scintillator Anti-Neutrino Detector at the Kamioka site. KamLand, pp. $1-121$

12. Dye ST (2012) Geoneutrinos and the radioactive power of the Earth. Rev Geophys 50(3): RG3007.

13. L Ludhova, Zavatarelli (2013) Studying the Earth with Geoneutrinos. Advances in High Energy Physics 2013: 1-16.

14. Geoneutrino Wikipedia.

15. L Ludhova, Zavatarelli (2013) Studying the Earth with Geoneutrinos. Advances in High Energy Physics 2013: 1-16.

16. TI Banks,SJ Freedman, J Walling, N Ybarrolaza, A Gando, et al. (2015) A compact ultra-clean system for deploying radioactive sources inside the KamLAND detector. Nucl Instrum Meth A7 69: 88-96.

17. Medical Engineering Students Training Center for Quality Control, Calibration, Repair, Maintenance and Use of Medical Equipment with Medical and Health Magazine.

18. The Electromagnetic Spectrum

19. Molecular Imaging Wikipedia.

$\begin{array}{ll}\text { BIOMEDICAL } & \text { Assets of Publishing with us } \\ \text { RESEARCHES } & \text { - Global archiving of articles } \\ & \text { - Immediate, unrestricted online access } \\ \end{array}$

